\title{
RETROSPECTIVE STUDY OF OPHTHALMIC CARE IN HIGH PROFILE PATIENT SCENARIO
}

\section{Ophthalmology}

Dr. Varshav Gore

Professor, Department of Ophthalmology, MGM Medical College and Hospital, Navi Mumbai

\begin{tabular}{ll}
\hline $\begin{array}{l}\text { Dr. Mamta } \\
\text { Agrawal* }\end{array}$ & $\begin{array}{l}\text { Junior Resident, Department of Ophthalmology, MGM Medical College and Hospital, } \\
\text { Navi Mumbai *Corresponding Author }\end{array}$ \\
\hline $\begin{array}{l}\text { Dr. Ayushi } \\
\text { Choudhary }\end{array}$ & Junior Resident Department of Ophthalmology, MGM Medical College and Hospital, \\
\hline
\end{tabular}

\section{ABSTRACT}

BACKGROUND VIP syndrome is a condition in which high profile people are shown to experience adverse treatment effects even in the hands of skilled physicians or surgeons in absence of any other potentially complicating risk factor. High profile patients can be categorized into three groups: Public figures, Relatives, and Colleagues and other doctors. Since ophthalmological surgeries are micro surgeries requiring precise measurements and techniques, any secondary influence over the surgeon's decision making powers, due to the presence of elite patients, has an undesirably affect. This is a retrospective analysis done after the surprising finding that correlates with many of my colleagues and we came to common conclusion about difference in approach towards known and unknown patients. METHOD 100 senior doctors belonging to different departments of a tertiary health care were inquired about their patients and the complications they faced while treating high profile patients by filling a questionnaire on the basis of their experience. RESULTS $90 \%$ of the doctors do categorize their patients into high profile patients, $80 \%$ of these doctors who faced complications had changed their routine treatment protocol. $93 \%$ of the doctors were cautious while treating these patients. $98 \%$ of the doctors agree with the bias present while treating high profile patients and consider the need to highlight VIP syndrome. CONCLUSION It is important to follow routine fixed protocol, no using new instruments or techniques and not to categorize any patient as high profile, manage such patients cautiously but without getting overcautious and maintaining confidentiality and privacy

\section{KEYWORDS}

Fixed protocol, High profile patients, Media pressure, Routine investigation, VIP.

\section{INTRODUCTION}

Doctor-patient relationship is the heart of practicing medicine. It forms the pillar of medical ethics and healthcare system. Every patient entering the hospital is important and requires the best possible care and treatment. However, it has been noticed that some patients get special treatment in hospitals in terms investigations offered, treatment provided and overall management proffered. The exact definition of a high profile patient cannot be made, as it changes from situation to situation and setup to setup. Special attention is given to people who might be known to the treating doctor or any staff of the hospital, any celebrity or public figure, any relative, doctor, or their colleague. When the person is known to you or society where fame can pressurize, disturb or distract the treating doctor into bad medicine. ${ }^{[1]}$ All the skilled and experienced doctors around the world have come across this similar issue while treating such High profile or VIP patients, so we decided to highlight this phenomenon of "VIP SYNDROME".

\section{Categories of high profile patients}

In an ophthalmic setup, we come across the following categories of high profile patients:

- Public figures- Public figures are people belonging to political background, business leader, celebrity, social media personality whose life and behavior is of public interest and is under public scrutiny .Celebrities and other prominent public figures bring the hospital into limelight and create stress for the treating doctors and treating staff. The overall responsibility of the doctors and staff increases for public figure patients due to the media exposure. Routine staff members are not used to dealing with the media on a daily basis, creating an environment of anxiety amongst them. This leads to change in the standard procedures and care system ${ }^{[2]}$ The staff is extra cautious and stressed. This deviation from the routine practice is not a good idea and it leads to unforeseen complications. This then induces a "vicious circle of VIP pressure and staff withdrawal". ${ }^{(3)}$

Besides this, the doctor in order to reduce the pain and come out with better results in such patients, tries to increase or decrease the number of investigations that are done in the routine workup, which later on might lead to unknown and unusual complications. ${ }^{[2]}$ Similarly, at times these celebrities request for unwise treatment and tend to cause pressure on doctors to let go invasive tests or deny to share medically relevant information with the staff in order to preserve their public image.

Minor hiccups in the treatment, which as common and uneventful become noticeable in such patients due to the public eye and create unnecessary havoc for the doctor and attending staff. Apart from all this, it becomes more difficult for the doctor to maintain confidentiality in such patients. In such a case, it is advisable to give the treatment and progress updates to only the person next to kin (like the spouse) and the senior advisor in the family.

- Relatives- When it comes to your own blood relations, the hands of the most skilled doctors also shake. Excellent experienced doctors also become anxious which may or may not affect the treatment but does disturb their mental peace which is noticeable and worrisome. In giving the best treatment, doctors tend to bend the rules and then can end up with complications. For example, not doing a dilated fundus examination, which is important and is a part of routine examination to know any retinal complications before cataract surgery. Change is not good in such cases because if it meant to do any good, it would have been the gold standard treatment modality.

For example, a renowned vitreo-retinal surgeon who had treated many central serous retinopathy (CSR) cases without complications, when had to treat his own brother in law for CSR landed up with subretinal neovascular membrane (which is a known but rare complication) after laser

- Colleagues and other doctors- Doctors are known to be the worst patients. They often tend to interfere and question the normal operating protocols and create anxiety for their attending medical team which might be competent but junior to them. Also, the treating doctor tends to be overcautious while treating such patients, which proves to be counterproductive maximum number of times. They also decide their own follow up dates and tend to report late or according to their will and wish.

\section{METHODOLOGY}

OBJECTIVE 
The aim of this study is to bring to light the importance of following standard procedures while treating all patients including high profile patients. These patients are found to be at an increased risk of adverse treatment effects and complications even with the best possible healthcare options and experienced physicians and surgeons. This may possibly be due to distraction of the healthcare team and deviation from the standard normal operating procedures.

\section{METHOD}

A total of 100 senior doctors belonging to different departments of a tertiary health care were inquired about their patients and the complications they faced while treating high profile patients. They were asked to fill a questionnaire (figure 1) on the basis of their experience. Consent was obtained from all doctors.

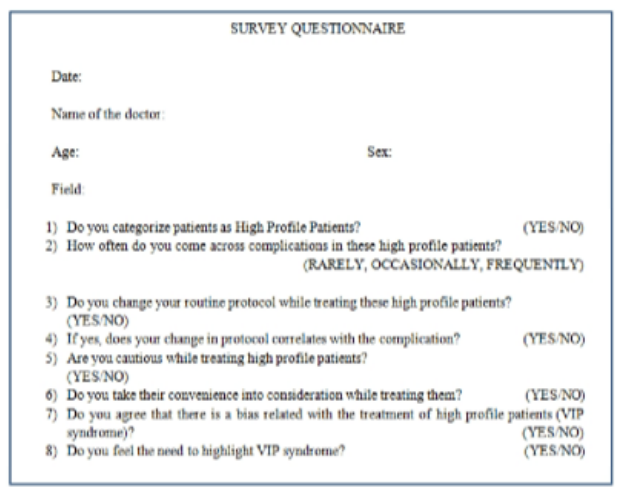

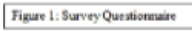

\section{RESULT}

Data analysis showed a significantly higher number of complications in the high profile group of patients which is medically unexplained. On the basis of the survey, we noticed that about $90 \%$ of the doctors do categorize their patients into high profile patients. $10 \%$ of these patients rarely show any complications, $60 \%$ occasionally develop complications, and $30 \%$ often land up in complications which remain medically unexplained. When asked, it was found that $80 \%$ of these doctors who faced complications had changed their routine treatment protocol in these high profile patients. $66 \%$ of the complications that occurred correlated with the change in the protocol. $93 \%$ of the doctors agreed that they were cautious while treating these patients. $95 \%$ of them also took patients convenience into account while treating them. $98 \%$ of them agree with that there is a bias related with the treatment of these patients and $98 \%$ of them also agree with the need to highlight VIP syndrome.

\section{(Figure 2)}

\section{DISCUSSION}

Ophthalmic surgeries are considered to be one of the finest microsurgeries of the world. Since the surgery drastically affects the visual sensations, any minor errors are conceived as unendurable by the patient. Hence the ophthalmologist must bear in mind to avoid even the slightest of the mistakes. This requires precise measurements of the eye, correct choice of implantable lenses and appropriate decision regarding the surgical techniques tailored to the individual patient needs. The treating surgeon is the best person to make these decisions as he has the complete and thorough knowledge regarding the case and can appropriately modify the treatment line to suit the patient's needs. However, treating high profile patients affect these decisions of the surgeons as they are overwhelmed with the need to satisfy these elite patients and often second guess their judgments. They deter from their tried and tested norms to satisfy these patients and every so often make wrong decisions

After a detailed analysis of the treatment given and the complications that had occurred in normal and high profile patients, we come to a conclusion that incidence of VIP syndrome is seen in fairly good number of patients in all the medical fields being treated by skilled physicians and surgeons. In order to reduce the incidence of VIP syndrome while managing high profile patients, certain institutes and hospitals have made guidelines for the same. [3] While there is no certain way to completely remove the bias associated with treatment of high profile patients, the author recommends a few practices that can ascertain avoidance of unnecessary risks and complications while simultaneously ensuring exceptional management of any category or type of high profile patient-

\section{1) Always follow the standard operating protocol (SOP)}

Any institute, big or small has a certain set of operating procedures and protocols in place. These rules are made by practiced clinicians, doctors and various ancillary members of hospital with the aim to provide the best healthcare to one and all patients and also ensure a smooth working of the hospital as such. Any changes or bending of these rules disrupts the normal smooth machinery of the hospital system leading to road blocks on the way. While the doctor may consider the bending of rules as a short cut or even a necessity, we need to realize the fact that changing protocols may invariably lead to complications that might in turn harm the patient or even, in rare cases, create problems for the doctors themselves. The circumventing of administration also leaved the doctor more vulnerable to personal disadvantage later on.

A very well-known example, though non ophthalmological, example of such a case, is the case of Anna Roosevelt, the First Lady of the United States of America, who was misdiagnosed with aplastic anemia before a complete workup was carried out to support such a claim. It was later found out that she suffered from military tuberculosis, alas timely treatment for which wasn't given and she passed away.

\section{2) Communication Vs. Privacy}

It is the duty of not only the doctor but the whole hospital team involved with managing the patient and its care to create a balance between communication with the patient and the next of kin while simultaneously ensuring that the patient privacy is maintained. This is necessary to ensure that all medically relevant information is communicated thoroughly. If the doctor or any other member of the team is star struck, the involved patient or even the family might be hesitant in sharing medical history. Similar might be the case if the people involved fear that the information they provide might lead to chatter. The doctor often might not discuss about possible complications or facts about prognosis in such scenario. The doctor should create confidence with the patient and relative team that ensure them that no information, private or otherwise would breach the doctor-patient confidentiality and become a source of news. This is necessary as any information, not shared could lead to further complications in patient management

For example, a patient $\mathrm{X}$ visited the ophthalmology department, of a tertiary care center with the complaint of blurring of vision and blue tinged vision. A detailed workup was carried out for the patient on a priority basis, still no cause could be pin pointed. It was later found out that the patient was taking tablets containing Sildenafil; however avoid mentioning so due to the associated embarrassment. The treating doctor also failed to ask him relevant questions because of an assumed impossibility of such a situation.

Special arrangements, if needed, should be made to keep such patients out of the limelight to avoid such miscommunication that hampers treatment.

\section{3) Avoid CHAIRPERSON'S/ CHIEF SYNDROME}

On admission of a VIP patient, the whole hospital including the management gets involved. It is assumed that chairperson i.e. the top $\mathrm{man} /$ chief is the best doctor in the institute [4]. But in reality, it is the treating doctor who has detailed knowledge about the patient and can handle the patient the best. The advice from the chairperson or even family doctor of the patient can be taken into consideration but the final decision making power should be with the treating doctor as he knows the best about the concerned patient.

\section{4) Resist anxiety}

Anxiety issue often occurs when you are treating your own relatives. 
Trying too hard only complicates things. Being nervous, overcautious or tense hampers your skills. For example, a nervous sportsman fails to keep up with his own capacities and expectations. Trying to do heroics in terms of medicine to give better treatment and impress the high profile patient, might turn out really bad for the doctor. It is important not to use any new instruments, machines, techniques which the doctor is not sure about or has not used before, since half knowledge is dangerous.

\section{5) Control media pressure}

High profile patients come with a lot of media pressure which puts doctor and staff into a lot of stress. Media demands exhibition of the treatment. Media wants to know everything about the diagnosis, treatment and prognosis in these patients. But it is responsibility of the treating doctor to maintain confidentiality. Zero information to the media might also lead to difficulties so a line between the two needs to be maintained. [3]

\section{6) Avoid Exhibition of treatment}

Live videos of the surgery or too many relatives in the room while a procedure is being done by the doctor can make the doctor conscious and may hamper his skills, so such a scenario should be avoided by allowing minimum relatives to enter the procedure room.

\section{7) Compliance}

High profile patients often turn out to be less careful and alert about their treatment. They rely more on the doctors. They also tend to have unrealistically high expectations from the doctor with less compliance from their side. They tend to have a casual approach in terms of follow ups, proper dosing, maintaining medical records etc. It hence becomes more important to convey them how important self-care is. Often these patients complain of minor problems which are not significant.

For example, a patient $Y$ always came with complaint of watering after a cataract surgery with no relevant anterior segment findings. Repeat thorough examinations were carried out and multiple treatment modalities were tried, still the patient had no relief.

We hereby conclude that,

1. Do not categorize any patient (label any patient as High profile/VIP).Treat them all equal.

2. Follow routine fixed protocol.

3. Don't try any new techniques to impress patients.

4. Don't use new machines.

5. Not to use any new instruments.

6. Don't skip any routine investigation or evaluation.

7. Value your medical knowledge and skills and be confident about it.

8. Manage such patients cautiously but without getting overcautious.

9. Maintain confidentiality and privacy.

\section{Conflict of interest: Nil}

Funding: No funding

\section{REFERENCES}

1) Groves, J., Dunderdale, B. and Stern, T. (2002). Celebrity Patients, VIPs, and Potentates. The Primary Care Companion to The Journal of Clinical Psychiatry, 04(06), pp.215223.

2) Block, A. (1993). Beware of the VIP Syndrome. Chest, 104(4), p.989.

3) Guzman, J., Sasidhar, M. And Stoller, J. (2011). Caring for VIPs: Nine principles. Cleveland Clinic Journal of Medicine, 78(2), pp.90-94

4) Blackburn, S. (2016). With quality and safety for all: Risking optimal care with VIP patients. Journal of Clinical Oncology, 34(7 suppl), pp.142-142. 